\title{
RECENSIONES
}

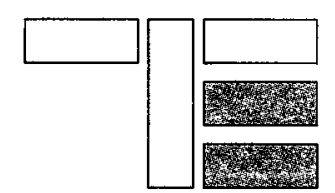



ABDALLAH-PRETCEILLE, M. (2001) La educación intercultural. Barcelona, Idea Books.

La presente obra ofrece dos partes diferenciadas; la primera referida a la elaboración de una teoría de la interculturalidad; la segunda se centra en la descripción de la práctica de la educación intercultural en Francia y en otros países como Suiza, Quebec y Alemania, así como en una propuesta de educación cívica, como eje central de una educación intercultural, para la construcción de la ciudadanía europea.

La primera parte tiene un gran interés: ofrece un análisis riguroso de los términos multiculturalidad e interculturalidad desde los contextos sociales en los que se les atribuye el significado, los ámbitos anglosajón y francés; y presenta las bases epistemológigas, metodológicas y éticas en las que se fundamenta la opción política y educativa de la interculturalidad.

En el primer capítulo, la visión que presenta la autora sobre la cultura en las sociedades contemporáneas es lúcida y sugerente. Defiende que cada cultura está en continuo movimiento y lo importante de ella son aquellos rasgos culturales que ofrece en cada momento, como ofertas de socialización y aprendizaje, a quienes pertenecen a ella por nacimiento o se acercan a ella por proximidad y contagio. La cultura de cada pueblo es reflejo de la diversidad de su mundo de la vida y no se deja aprisionar en estructuras formales y rígidas. La diversidad y la diferencia aparecen tanto en el interior de una cultura como en el interior de las sociedades conformadas por culturas plurales. Por lo

(c) Ediciones Universidad de Salamanca tanto, la reflexión sobre la identidad de una cultura implica la reflexión sobre la diversidad cultural y sobre los problemas relativos a la diferencia.

En el segundo capítulo hace un análisis de las insuficiencias del modelo multicultural, presente en la tradición anglosajona, en sus principios y postulados: prioridad al grupo de pertenencia, localización espacial de las diferencias, una jurisdicción específica y compleja que garantice los derechos de cada cual, el reconocimiento del relativismo cultural y la expresión de las diferencias en el escenario público. También analiza las consecuencias prácticas de tal modelo: acentuación de las actitudes de rechazo y exclusión, limitación de la movilidad social y el encubrimiento del carácter cada vez más policromo y polimorfo de los grupos y de las culturas.

Martine Abdallah-Pretceille apuesta por la interculturalidad si se quiere construir verdaderas comunidades sociales: abiertas a todas las formas de minoría, en las que se considere tanto a los individuos como a los grupos, donde la pertenencia sea consecuencia de la filiación y no de la procedencia, y se acentúen los valores universalistas o comunes a unos y a otros.

Lo más novedoso de la obra, a mi juicio, es el planteamiento epistemológico que se hace en el capítulo tercero: "Lo intercultural se fundamenta en una filosofía del sujeto, es decir, en una fenomenología que construye el concepto de sujeto como un ser libre y responsable, inscrito en una comunidad de semejantes. El enfoque intercultural rompe con el punto de vista objetivista y estructuralista, ya que su interés principal es la 
producción de la cultura hecha por el mismo sujeto y las estrategias que usa" (p. 39). De acuerdo a tal planteamiento, el eje metodológico del análisis intercultural tiene que centrarse en el desarrollo de la comprensión de las situaciones sociales, la aplicación del interaccionismo simbólico para indagar cómo conciben los sujetos los elementos culturales de una situación dada y la adopción por el investigador de una perspectiva situacional y complementaria. La autora también plantea la interculturalidad como un proyecto ético, fundado sobre las siguientes bases: la dignidad de cualquier otro y su reconocimiento como un igual, la búsqueda del acuerdo y de la solución de los conflictos, la vigencia de los derechos humanos y la laicidad de la sociedad.

La segunda parte del libro, que me parece de un interés menor, responde probablemente a la preocupación de la autora por orientar las acciones educativas que, en definitiva, traducen la práctica de las opciones filosóficas y éticas que ha defendido en los capítulos anteriores.

Me parecen sugerentes las propuestas sobre la educación cívica para la construcción de la Unión Europea como una comunidad de ciudadanos. La autora considera que en una sociedad plural, la apuesta por la educación cívica es doble. Por una parte, se trata de instalar los valores democráticos y los valores comunes en una sociedad cada vez más heterogénea. Por otra, se trata de pensar en el vínculo cívico con arreglo a una pluralidad de fidelidades y no a partir de la unicidad y de la exclusión. La identidad europea no excluye la identidad nacional, ni tampoco esta última la identidad regional o cualquier otra fórmula de afirmación de la identidad.

La educación cívica para una Europa intercultural, además de los valores éticos señalados en la primera parte, tiene que promocionar las actitudes de descentración de la propia cultura y de empatía hacia las gentes y culturas diversas, el control de los prejuicios y estereotipos, y las competencias comunicativas que permitan el conocimiento de los otros y la reflexión sobre uno mismo y el propio grupo. El presente libro aporta conceptos, propuestas teóricas y orientaciones para quienes están interesados en la educación intercultural.

JuAn Escámez SÁnchez

CASARES GARCÍA, P. M. (2002) Ancianos. Problemática y propuesta educativa. Madrid, San Pablo.

El envejecimiento de la población es uno de los hechos demográficos más característicos de las sociedades europeas actuales. España no es una excepción: el porcentaje de personas mayores ha ido aumentando al ritmo de los avances de la medicina y del descenso de la natalidad. Desde luego, esto es un signo de progreso - al menos de progreso material-, sin embargo, también plantea problemas a los que es necesario dar respuesta. Esta búsqueda de alternativas es, sin duda, uno de los propósitos del texto que comentamos.

Plantear soluciones ante determinadas circunstancias requiere, en primer lugar, definir y describir, de ahí que en la primera parte, la autora, tras aportar unos interesantes datos sobre 
el porcentaje de ancianos en nuestro país - desde la década de los noventa hasta la previsión para el año 2050-y sobre el incremento de la esperanza de vida, abra paso a la pregunta: "¿Qué es ser anciano?".

Para explicar la ancianidad, los factores son numerosos. La edad no es un criterio definitivo ni siquiera suficiente. Ser anciano es un momento de cambios -físicos, biológicos, intelectuales, estéticos- distintos en cada persona que hacen de la vejez una edad crítica que requiere adaptación. A ello se unen condicionantes histórico/sociológicos que repercutieron en el estatus de los mayores, el momento de la jubilación, pérdidas en el mundo de las relaciones personales, problemas de comunicación, el limitado concepto social respecto a la sexualidad de los ancianos, el aislamiento, la exclusión —a veces autoexclusión - y el comienzo del camino hacia la "cuarta edad".

Las variables anteriores están relacionadas con los problemas sentidos por las propias personas mayores, tomados de fuentes tan vivas como el Téléfono Dorado y el Teléfono de la Esperanza. En ellos se perfila la soledad como uno de los sentimientos carenciales más fuertes.

La soledad de los ancianos, su aislamiento social, procede en gran medida de prejuicios que crean hacia ellos -o en ellos mismos- actitudes negativas hacia la vejez. Esta situación se explica, en parte, por las características de la sociedad posmoderna; en parte, por la imagen de la ancianidad que transmite la acción educativa informal, desde la Biblia al uso del lenguaje, el refranero, los medios de comunicación social (anuncios, películas, tebeos, canciones). A ello se une que, en el marco familiar, no siempre se encuentra una educación adecuada de cara a la vejez; y otro tanto puede decirse de la escuela: pese a que el tema de la vejez ha comenzado a introducirse de modo incipiente en los diseños curriculares es aún uno de los grandes olvidos del sistema educativo formal.

Planteada la situación y posibles causas que la generan, la segunda parte del libro presenta respuestas sociales a los principales problemas en tres niveles: mundial (las acciones de la ONU y de la OMS), europea y española. Esta última aborda luces y sombras de las pensiones y la sanidad; una amplia y orientadora relación y descripción de servicios sociales; participación y asociacionismo; ocio y cultura; administraciones públicas y políticas sociales.

El tercer bloque de contenidos, bajo el título "Sugerencias y pistas", presenta otras alternativas de acción dirigidas a la escuela, a la familia, a las empresas y a los propios mayores, quienes, a lo largo del texto, se han ido perfilando progresivamente como ciudadanos activos que constituyen un importante un capital humano y que continúan ofreciendo su aportación a la sociedad.

El trabajo se completa con un directorio en el que se recogen servicios sociales, ayuda telefónica, asociaciones, voluntariado, programas universitarios para mayores, prensa, revistas, programas radiofónicos y páginas WEB dirigidas a personas mayores o creadas por ellas.

Con un planteamiento decididamente educativo, que invita a la reflexión, el texto que presentamos constituye un 
trabajo bien documentado que compagina el rigor de un estudio serio y la utilidad con un lenguaje ameno, sencillo y directo que lo hace accesible a todos los lectores.

ANDRÉs SORIANo DíAZ

COLOM CAÑELLAS, A. J. y NÚÑEZ CUBERO, L. (2001) Teoría de la educación. Madrid, Síntesis.

Hay libros que superan los objetivos trazados por sus autores. La obra Teoría de la educación de los profesores A. J. Colom Cañellas y L. Núñez Cubero es buena prueba de ello. La lectura y estudio de este libro, que surge con vocación de manual universitario, responde no sólo a la finalidad teórico educativa para la que ha sido redactado; esto es, responder a las pretensiones praxiológicas, o mejor aún, tecnológicas, de toda teoría que halla en la acción educativa y los "universos educativos" donde ésta se desarrolla su posibilidad de ser construida con rigor y exhaustividad, sino que también satisface otros objetivos a los que, colateralmente, sus autores han sabido responder con acierto sin que hayan sido dichos objetivos su preocupación fundamental. Me refiero al intento, claramente logrado, de formalización de una "nueva" Teoría de la Educación centrada en las nuevas necesidades de conocimiento y de aprendizaje que demanda hoy la sociedad cognitiva. Aunque los autores insistan en la idea que la pretensión del libro no sea otra que la actualidad y no la novedad (p. 10), en realidad, los lectores hallarán en él un enfoque renovado de la Teoría de la Educación además de unos conocimientos teórico educativos plenamente actualizados.

Esta renovada Teoría de la Educación que sistematizan los autores se apoya, desde el punto de vista metateórico o paradigmático, en la nueva teoría sistémica, estructurada sobre la base de la teoría de la complejidad y el constructivismo psicológico. De este modo, las clásicas cuestiones que se abordan desde la Teoría de la Educación - la educación y la práctica educativa, prerrequisitos, características y elaboración de la acción educativa desde la perspectiva del educador y el educando, la evaluación, entre otros aspectos analizados- se impregnan de una nueva lectura paradigmática que encuentra, como sus propios autores indican, en la "libertad cognitiva y moral" del educando y en la dimensión innovadora del conocimiento versus la naturaleza acomodaticia del saber y la información transmitida en los contextos instruccionales, la base de su legitimidad como teoría educativa.

De ahí que no resulte extraño que en los capítulos que desarrollan los fundamentos teóricos de la práctica educativa - tercera parte de la obra-, la centralidad de los temas abordados se ocupan más de establecer normas y estrategias orientadas a activar ciertos procesos psicológicos fundamentales en todo aprendizaje (por ejemplo, la motivación), sin los cuales, la actividad educativa aparecería completamente vulnerable al propio educador. Y que, del mismo modo, tampoco resulte extraño que esta Teoría de la Educación articule una nueva "ingeniería pedagógica", de corte constructivista, orientada 
a desarrollar los procesos cognitivos básicos - atención, memoria, percepción- involucrados en los aprendizajes complejos (comprender, razonar, interpretar, argumentar, resolver problemas, etc.) y establecer pautas que ayuden a los docentes y educadores en general a "enseñar a pensar" y lograr entre sus estudiantes el principio educativo actual de "aprender a aprender" que es donde, en definitiva, se resuelve la eficacia de las acciones educativas en el nuevo marco de la sociedad cognitiva.

La obra se estructura en tres partes bien diferenciadas articuladas, cada una de ellas, desde la visión tecnológico-sistémico-constructivista de la práctica educativa. La primera parte, se dedica al estudio de Los aspectos teóricos de la educación. El capítulo primero aborda la educación desde el paradigma de la complejidad y la interpretación sistémica - que no holísticade la misma. Los autores introducen al lector en las teorías de la complejidad de Edgar Morin y Jöel de Rosnay y proponen, a partir de los supuestos teóricos en los que se apoya dicho paradigma, incorporar esta visión a la hora de entender y explicar la educación a partir de un conocimiento analítico-sintético de la realidad educativa. El posicionamiento epistemológico de los autores se expresa, de modo claro y contundente, en el apartado que dedican a la cuestión paradigmática en educación. La denostada y criticada visión tecnológica de la educación presenta en esta renovada Teoría de la Educación una interpretación epistemológica del término tecnología. De este modo, el conocimiento tecnológico —o lo que es lo mismo, lo tecnológico- como bien señalan los autores "no es un paradigma, sino la característica esencial de los tres paradigmas" educativos. Lo tecnológico se refiere a un tipo de conocimiento que tiene como objetivo la producción o transformación de una realidad -en nuestro caso, educativa- de modo intencional. Esta acepción de lo tecnológico se vincula con el significado epistemológico que se atribuye en la Filosofía de la Tecnología al propio término "tecnología"; esto es, sistema de acciones intencionales con vistas a la realización de objetivos y metas que desean lograrse. En este sentido, la educación - quiérase o no-, es tecnología, del mismo modo que el conocimiento tecnológico es el tipo de conocimiento que requiere dicha "tecnología" (léase, dicha práctica educativa). Y ello es válido para la totalidad de los paradigmas educativos, independientemente de las orientaciones ideológicas o científicas a las que dichos paradigmas se adscriban.

El segundo capítulo de esta primera parte - Acerca de la Teoría de la Educación"- lo dedican los autores a analizar el recorrido histórico de la Pedagogía y el nacimiento y evolución de la Teoría de la Educación en el contexto académico y universitario en España. Su lectura explica algunas sinrazones o irracionalidades de los que somos testigos en algunos contextos universitarios. El último capítulo de esta primera parte analiza, con rigor, unos de los elementos básicos que da sentido a toda acción educativa: la cuestión de las finalidades y de los valores en educación. Especial interés reviste la crítica que hacen los autores a la escuela pluralista a la que opondrían 
una escuela más democrática y plural, basada en la excelencia, la equidad y los derechos humanos promotora, al mismo tiempo, de la tan anhelada cohesión social.

La segunda parte del libro se centra en "el estudio de la acción educativa" de la que forman parte los capítulos cuatro y cinco. En él se sintetizan los prerrequisitos básicos de la acción educativa desde la perspectiva del docente, el aprendiz y la fase preactiva de la enseñanza; esto es, la planificación del currículum y los niveles y tipologías curriculares existentes.

La tercera y última parte de la obra - Fundamentos teóricos para la práctica educativa - reúne cinco capítulos que ofrecen al lector los objetivos praxiológicos propios de la visión teórico práctica de la Teoría de la Educación de la que parten los autores. Organizados bajo la interpretación cognitivo-constructivista de la teoría de la información y la comunicación formativa, los capítulos 6,7 y 9 se ocupan respectivamente de los tres procesos básicos implicados en todo sistema de comunicación con finalidad formativa (o educativa). De un lado, los procesos de emisión en la escuela (capítulo 6), aplicables, por lo demás a cualquier otro contexto no formal de educación, en la que los autores hacen acopio del acervo de teorías psicológicas de la motivación para sistematizar, desde la Teoría de la Educación, un sistema pedagógico que aborde cómo encarar los procesos de motivación en el aula desde cada uno de los marcos teórico psicológicos vigentes (conductual, psicosocial, atribucional, constructivista, etc.). De otro, se abordan asimismo los

(C) Ediciones Universidad de Salamanca procesos de recepción y de estructuración de la información para la formación (capítulos 7 y 9) para propiciar justamente el potencial innovador del conocimiento y, por ello, la creatividad y autonomía cognitiva del estudiante. En estos dos capítulos, las transferencias pedagógicas que realizan los autores se realizan a partir del constructivismo, lo que no evita que para llegar a ello, el lector encuentre abundante material ofrecido por otras teorías cognitivas desde las que encarar en la práctica las estrategias cognitivas de los estudiantes habida cuenta de la importancia que éstas tienen en los procesos superiores de aprendizaje.

Por último, los capítulos 8 y 10 se encargan de analizar los agentes mediacionales de la educación y la evaluación educativa. En el primer caso, se especifican las acciones y criterios de acción coherentes con los distintos modelos propuestos desde la psicología para encarar los procesos comunicativos por los educadores (aprendizaje observacional de Bandura, aprendizaje social de Bruner, modelo neoconductual de Gagné). Al mismo tiempo, se describe la diversidad de métodos en educación con un breve análisis de la evolución de los mismos a partir del movimiento de renovación pedagógica de la Escuela Nueva. De especial interés el apartado que dedican los autores a los ambientes educativos o, lo que es lo mismo, los espacios físicos donde la acción educativa tendrá lugar y a la propuesta tecnológica del aprendizaje en los formatos educativos a distancia basada en similares propuestas que desarrollan, por otra parte, los autores en el capítulo que dedican a la información para la 
formación; es decir, al diseño de una tecnología (o ingeniería educativa) metacognitiva del aprendizaje aplicada, en este caso, a la educación a distancia. La centralidad del capítulo 10 se dedica a la evaluación educativa que, como sus propios autores indican, será abordada desde la perspectiva de los agentes de educación y no desde la perspectiva de las técnicas y procedimientos de recogida de datos. Esta visión, que coincide, por otro lado, con la visión teórico educativa de la evaluación, ayuda al lector a introducirse y recorrer los distintos paradigmas o enfoques que han dominado y, aún hoy, siguen dominando - aun cuando se los critique o rechace- en la práctica educativa. Conjuntamente con el modelo tradicional de evaluación - la evaluación sumativa-, los autores desarrollan los posteriores enfoques que jalonan en la actualidad la evaluación educativa. De un lado, el modelo operacional de evaluación ("pedagogía de la curva en j"), que se propuso para la Reforma educativa de 1970 —evaluación formativa conductual-, a los actuales modelos -más en el plano teórico que en su aplicación práctica por el profesorado y otros agentes evaluadores- de evaluación formadora y la evaluación comunicativa o progresiva inspirada en la visión constructivista y sociocultural del aprendizaje.

En definitiva, estamos ante una obra en la que los investigadores en educación, estudiantes y los profesionales de la educación - pedagogos, educadores sociales, formadores, psicopedagogos, profesores - encontrarán una información indispensable para su quehacer profesional cotidiano, que sistematiza con rigor y sencillez las teorías científicas que legitiman y hacen posible la racionalidad científica de su hacer, a la par que hallarán en este libro la lectura "teórico educativa", es decir, teórico práctica, de dichas aportaciones científicas bajo la forma de criterios, pautas y estrategias para el diseño, elaboración y puesta en práctica de la acción pedagógica. La dilatada trayectoria académica y científica de los profesores A. J. Colom y L. Núñez — catedráticos de Teoría de la Educación de las Universidades de las Islas Baleares y Sevilla respectivamente - suponen además un referente de sobrada garantía como para afirmar que nos encontramos ante un riguroso y actualizado trabajo del que podemos asegurar que se trata de una obra innovadora, clarificadora y contundente que arroja una nueva luz para abordar la complejidad de la práctica educativa.

Clara Romero Pérez

GARCÍA CARRASCO, J. y GARCÍA DEL DUJO, A. (2001) Teoría de la Educación. Procesos primarios de formación del pensamiento y la acción. Salamanca, Ediciones Universidad.

Han transcurrido cuatro años desde la publicación de lo que podríamos llamar primera incursión de un amplio proyecto de trabajo sobre los problemas básicos de la educación. Y decimos amplio proyecto porque tal como se enunciaba en el primer volumen, el ámbito de la Teoría de la Educación asumía como subtítulo particular el 
siguiente: "Educación y acción pedagógica".

En este caso lo que se intentaba era ofrecer una primera reflexión sobre el fenómeno educativo al mismo tiempo que se realizaba una aproximación al nacimiento de la pedagogía, las cuestiones básicas de la epistemología a través del estudio de la problemática cognoscitiva de la construcción de una teoría de la educación para concluir con el estudio de la acción pedagógica desde su "anatomía y fisiología" con la intención de encontrar aquellos rasgos que permitan interpretarla como acción tecnológica.

Pero ya en este primer volumen los autores nos enunciaban que su texto no se limitaba al ámbito, concepto de la educación y epistemología del discurso pedagógico. En su mente estaba no solo plantear qué cosa es la educación y reflexionar sobre ella sino afrontar un nuevo interrogante: ¿cómo somos para poder ser educables y qué acontece al educarnos?

Es este interrogante el que impregna las principales cuestiones del segundo volumen bajo el subtítulo de uprocesos primarios de formación del pensamiento y la acción".

Desde la primeras páginas nos anuncian el nuevo interrogante para su próximo trabajo: ¿qué es importante hacer para educarse? Centrándonos en este segundo volumen recién editado, podríamos decir que dentro de su estructura unitaria determinada por diez capítulos diferentes, se distinguen diversos contextos que sirven como guía para que el lector vaya caminando y comprendiendo el supuesto básico que lo fundamenta: los cambios en los sistemas de comunicación constituyen sucesos culturales de una categoría especial que caracterizan al ser humano: la intercomunicación.

En términos de los propios autores "el género humano, sigue siendo animal genuino, ser vivo genuino pero con una excepcional capacidact de elaboración de comunicación: de información acerca del entorno vital". Tal hecho conduce a una realidad particular de la especie humana que necesita de la cultura para vivir. Es una necesidad vital de nuestra especie frente a las otras especies animales.

Hasta llegar al capítulo que cierra este volumen, "Creación de cultura y proceso educativo", la obra expone lo que podríamos llamar presupuestos básicos en sus tres primeros capítulos. El primero de ellos dedicado a los "sujetos y sucesos de la educación" que concluye con una visión global de la educabilidad que difiere de los procesos de madurez fisiológica y de clesarrollo y radica fundamentalmente en la interdependencia funcional entre el individuo y su entorno. Un entorno no limitado al espacio, a técnicas y diseños curriculares para adquirir conocimientos sino también sobre otros planes y proyectos de políticas culturales locales, regiones y sobre los procesos de dinamización comunicacional entre individuo y medio, entre individuos, entre culturas.

Esta intercomunicación es un referente para tener en cuenta temas esenciales objeto de reflexión específica en cada uno de los otros dos capítulos. Uno dedicado a «a autonomía, reconocimiento, cooperación y subjetividad" que no se viven de una forma aislada 
sino en relación con el entorno. El otro preocupado por el estudio del entorno descle la perspectiva de la "ecopoiesis". Es decir, no como espacio meramente físico sino como un asunto entre seres vivos en una dinámica en la que está presente la conciencia reflexiva y la mediación del exterior que constituye su oportunidad de acción. El entorno es donde se realiza el proceso de socialización cuyos ejes básicos vienen determinados por la orientación de la conducta en función de normas sociales y participación en conductas cooperativas.

Este entorno como mediación para la construcción humana es un entorno analizado y considerado no de forma estática sino a lo largo del proceso de evolución y desarrollo humano que comienza con el lugar del hombre en la biosfera y concluye con las diferencias entre los seres humanos que no se reducen a la variación genética sino que asume otros rasgos cualitativos que se evidencian en el fenotipo en determinados ambientes y pueden ser producto de la historia personal y social o de acontecimientos de carácter físico o psicológico. Esta diversidad no exime de la igualdad de derechos y deberes. La desigualdad no es algo biológicamente dado ya que la naturaleza proporciona únicamente diferencias, sino socialmente impuesto.

El estudio de subjetividad, de la interacción con el entorno y de la evolución como elementos importantes en la construcción humana conducen a los autores a profundizar en dos temáticas ampliamente interrelacionadas: el cuerpo y la mente. El primero es el determinante tanto de la vida encarnada como de la comunicación corporal, de su "cultura

(c) Ediciones Universidad de Salamanca corporal y psicosomática" como también contexto y situación de algunos acontecimientos problemáticos: la enfermedad y el envejecimiento. En todo caso la enfermedad no puede considerarse únicamente desde el punto de vista predominantemente anatómico sino que debe insertarse en los objetivos de la formación.

En cuanto al envejecimiento su calidad depende mucho de la consideración que le otorguemos. Hoy está cobrando importancia en el discurso educativo para dar oportunidades específicas de formación a un colectivo cada vez mayor cuya finalidad no es la preparación y actualización profesional sino seguir disponiendo y utilizando los bienes culturales y disfrutando de los apoyos sociales que les otorguen mayor autonomía evitando su desvalorización.

El órgano de la mente relacionado tanto con la plasticidad y educabilidad consciente e inconsciente abre camino para explorar intervervenciones directas en la estructura cerebral. Los autores se posicionan críticamente frente a una sociedad psicocivilizada a través de intervenciones directas (agresión física, intervención quirúrgica, interferencia química o manipulación mental...) como situación que oponen a los criterios básicos de la formación: libertad de acción, orientación asumida, pensamiento consensuado, reflexión crítica sobre el comportamiento personal y colectivo.

En el contexto de esta actividad mental con sus posibilidades y también sus riesgos, destaca la especial relevancia otorgada tanto al problema de la inteligencia, al entendimiento social como al intercambio afectivo que componen un 
entramado básico del conocimiento e intervención educativa.

En el primer caso se analizan y critican las perspectivas más estrictas del programa cognitivista que desarrolla la metáfora del ordenador pero se olvida de que el trabajo educativo no puede prescindir de los significados, los aspectos sobre los que se focaliza la atención y los contenidos con los que la motivación se alimenta.

En cuanto al entorno social, merece especial atención la revisión crítica de los trabajos de Vigotsky y los actuales planteamientos sobre las funciones psicológicas para concluir que el desarrollo humano es imposible fuera del contexto social. Las carencias continuadas en este contexto (abandono, hospitalismo prolongado, violencia, etc.) llegan a producir efectos lesivos importantes.

Esta necesidad de interacción humana conduce al estudio del campo afectivo en el discurso pedagógico. Un tema que con frecuencia ha sido minusvalorado y no se le ha otorgado la importancia que merece. Por ello tras analizar los distintos aspectos del intercambio afectivo (categorías, tramas, procesos de formación de la aptitud emocional y cultura emocional), concluyen con una serie de sugerencias pedagógicas claves destacando, entre otras, el rol que juegan las emociones en la vida moral y en la regulación de la práctica comportamental de los individuos y grupos.

Si a lo largo de la obra se ha mantenido desde el principio que los seres humanos tenemos dos espacios vitales interdependientes, el de la vida "que compartimos" con los otros seres vivos y el de la vida "que construimos" incorporando la cultura, no podría faltar un apartado dedicado a las relaciones entre cultura y proceso educativo.

El proceso cultural como secuencia de formación es considerado desde el punto de vista de transferencia de información, de la acción comunicativa así como de los propios sistemas de comunicación que hoy, más allá de la función lingüística y la aplicación gráfica, permiten la creación de situaciones virtuales, la ruptura del tiempo y espacio y la categoría de distancia. Todo un cambio que transforma las estructuras y abre diferentes perspectivas generando nuevos contextos y situaciones en la denominada sociedad de la información.

A pesar de estos hechos que determinan la realidad actual, en sus páginas finales este segundo volumen no se olvida de reconsiderar la función esencial de la Teoría de la Educación ya planteada en el anterior trabajo.

Esta función se sintetiza en proponer como meta general opciones de "calidad de vida". Tal finalidad básica, dentro de su ambigüedad y borrosidad, asume una serie de elementos como tener criterio crítico, pensamiento complejo para la búsqueda de dicha calidad en lo particular y local donde se produce la acción y para ser espectador reflexivo de lo global.

Este reto utópico de toda acción intervención educativa consideran que es un quehacer fundamental y asumible aunque inacabado como toda construcción humana teniendo en cuenta diferentes dimensiones: lo intrasubjetivo y lo intersubjetivo, la fundamentación del comportamiento moral, la educación intelectual, la formación del pensamiento complejo, la iniciación en 
el campo científico-tecnológico, la reflexión sobre las instituciones de formación que promueven el programa de acción del sistema de enseñanza sin olvidar que dentro de las transformaciones culturales de la sociedad actual es necesario también ampliar la zona de libertad del tiempo dedicado a la actividad lúdica, la diversión etc.

En síntesis, este segundo volumen profundiza en aspectos más concretos del quehacer pedagógico: los denominados procesos primarios de pensamiento y acción. El lector se encontrará con una obra que ofrece una continuidad respecto al primer volumen con una mayor amplitud de temas por la intención ya manifestada desde el principio de interesarse tanto por la "autopoiesis" (integración de la subjetividad y personalidad autónoma del ser humano) como por la "ecopoiesis" (conjunto de procesos transaccionales que originan transformaciones en los organismos) que determina la importancia del entorno social en la construcción humana.

En cuanto texto pensado como manual universitario, creo que la visión comprensiva, sistemática y profunda que ofrece del proceso educativo en toda su complejidad le otorgan una relevancia importante no sólo por la originalidad de sus planteamientos sino por la organización sistemática y muy bien estructurada de sus contenidos.

Agustín Requejo Osorio
GENNARI, M. (2001) Filosofia della formazione dell'uomo. Milano, Bompiani.

Mario Gennari - professore ordinario di Pedagogia generale nell'Università di Genova - prosegue ora la linea di ricerca avviata con l'originale volume, del 1995, Storia della Bildung. Formazione dell'uomo e storia della cultura in Germania e nella Mitteleuropa. Infatti, il testo qui sub specie recensionis rispecchia l'esigenza di far penetrare il lettore nei mondi richiamati dal concetto di Bildung, assunto come il darsi e il prendere forma armonica dell'umano. Tuttavia, in Filosofia della formazione dell'uomo rilucono anche altri filoni di ricerca sviluppati da Gennari e di cui è difficile trascurare il rilievo, propedeutico rispetto alla trattazione di tale opera. Ad esempio, gli studi focalizzati intorno al processo interpretativo nel contesto pedagogico (cfr. Interpretare l'edu-cazione. Pedagogia, semiotica, ermeneutica, La Scuola, Brescia, 1992, già preceduto dall'opera prima Pedagogia e semiotica, La Scuola, Brescia, 1984) o le ricerche sugli ambienti sociali e educativi o sulla città ( $c f r$. Pedagogia degli ambienti educativi, Armando, Roma, 1988; Semantica della città e educazione, Marsilio, Venezia, 1995) introducono e "preparano" ulteriori sviluppi delle problematiche ad essi sottese.

Prima ancora di giungere alla prima parte di questo volume, chi si prepara a studiarlo è chiamato a soffermarsi sulle nitide pagine dell'Isagóge. Il titolo scelto per tale "introduzione" all'opera 
—ovvero Disumano, troppo disumanocompendia significativamente il pensiero crudo e autentico di Gennari nei confronti del Novecento. Egli scrive: "il volume $[\ldots]$ costituisce una lunga riflessione, ma anche una denuncia circostanziata, a proposito delle filosofie della formazione a cui la tarda modernità ha dato vita" (p. 17). Filosofia della formazione dell'uomo è invero sostenuto da una riflessione critica che è costantemente attraversata da due afflati fondamentali. Il primo si radica nella ricchezza inesauribile dell'umanità dell'uomo che prende forma e si transforma in ogni tempo e circostanza, in ogni luogo e àmbito di vita. Il secondo riprende una lotta mai sopita contro ogni forma di nichilismo: di annientamento dell'uomo, dell'umano, dell'umanità. Per questo, ad esempio, Gennari sceglie di non dimenticare mai la Shoah, dichiarando "contro" quali culture nichiliste vuole indirizzare la propria critica graffiante. Contemporaneamente, però, l'autore sottolinea che il suo testo è anche "per" le persone che hanno contribuito alla sua formazione, nonché per quei lettori capaci, senza pregiudizi di alcuna origine, di discutere e interpretare le sue parole con libertà e autenticità.

Pertanto, - letta l'introduzionechi si accinge a inoltrarsi nelle oltre ottocento pagine di Filosofia della formazione dell'uomo è consapevole che dovrà, a sua volta, compiere una scelta: una scelta pedagogica decisiva fra l'umanesimo e il nichilismo, l'essere e il nulla, l'umano e il suo annichilimento, la formazione nell'umano e la deformazione dell'umano. Tuttavia, l'autore non suddivide umanesimo e nichilismo trattandoli separatamente, poiché lo scontro deciso e la contrapposizione netta si sviluppano di continuo.

È chiara, fin dall'Isagóge, la posizione di Mario Gennari, che mai riflette ambiguità nelle scelte o si nasconde dietro altrui visioni del mondo. Il suo è un dialogo costante con il lettore e, ancor prima, con gli interpreti della Bildung e del Novecento. Con questi studiosi Gennari si confronta: permette loro di esprimersi in prima persona attraverso un frequente uso di citazioni, ma non li lascia soli. Intesse una discussione critica che sembra poter proseguire oltre le pagine del volume. La presenza dell'autore si può percepire in ogni passaggio, anzi talvolta si profila persino "provocatoria" poiché coinvolge e interroga il lettore costringendolo a sostare sulle parole e i loro significati profondi, sui concetti e la loro feracità categoriale, sulle dinamiche sociali, politiche e economiche che coinvolgono l'uomo contemporaneo.

La maturità logica e critica -fondata ed espressa in maniera limpida e precisa - manifestata dall'autore di Filosofia della formazione dell'uomo si riflette nella struttura del volume. Ogni parte in cui è articolato il testo non manca di richiamarsi alle altre, così come ciascun capitolo e ogni paragrafo. $\mathrm{Al}$ lettore è richiesto un requisito fondamentale: essere un cultore dell'uomo, a qualunque disciplina o scienza facciano capo i suoi interessi. L'articolato indice lo potrà guidare ora fra la filosofia del diritto e la filosofia della politica ora all'interno delle antropologie e delle poetiche della Bildung.

Teor. educ. 13, 2001, pp. 269-296 
Proprio sulla Bildung si concentra la prima parte del volume: "Categorie della Bildung". All'iniziale capitolo dedicato alla flessibile filologia e filosofia della Bildung segue il più ampio capitolo dell'intero volume. Esso interpreta le parole, ma soprattutto le concezioni della Bildung formulate da I. Kant, J. H. Pestalozzi, F. W. A. Fröbel e F. D. E. Schleiermacher. Non si tratta, però, della ricerca di una definizione del concetto di Bildung: Gennari non si limita a trascegliere la più significativa tra le idee di formazione, poiché la Bildung si arricchisce di molteplici sfumature. Inoltre, Gennari indica una via euristica non tradizionale: cercare l'idea di formazione dell'uomo nelle pagine di studi che considerano la criticità razionale dell'uomo o la sua etica, e poi la natura e l'esperienza, l'infanzia e il gioco, la religiosità e l'interpretazione. Rappresentano un felice esito i risultati raggiunti nell'analisi dei quattro studiosi di lingua tedesca, scelti non casualmente dall'autore. Tuttavia, la prima parte del volume non si esaurisce qui. La Geburt der Tragödie di Nietzsche, la Krisis di Husserl e il Brief über den "Humanismus" di Heidegger si presentano come ulteriori interlocutori del "principio di formazione", che però richiama ora la crisi dell'umanesimo. Quest'ultimo capitolo introduce la trattazione successiva.

Le profonde significazioni elaborate nella seconda parte di Filosofia della formazione dell'uomo non possono essere sintetizzate nel breve titolo "Modernità e stili formativi", poiché esse coinvolgono molteplici aspetti e temi atti a rintraciare nei termini "modernità" e "stili formativi" soltanto i loro poli di

(C) Ediciones Universidad de Salamanca riferimento. Quello di "stile formativo" rappresenta un concetto originale, affrontato in particolare nel quarto $e$ conclusivo capitolo della parte seconda. In esso la krasis della modernità schiude non soltanto l'idea di una "società satura", ma anche la problematicità di una proliferazione di stili formativi che richiama le retoriche della globalizzazione e del postmoderno, nonché i percorsi additati dal progresso e dalla civilizzazione. Ebbene, la modernità è analizzata fin nei suoi più celati recessi. La scienza e il linguaggio sono i primi elementi di confronto scelti da Gennari e letti attraverso la filosofia della scienza e la filosofia del linguaggio di Wittgenstein e Popper, Feyerabend e Kuhn. La metropoli e la moda costituiscono le successive "lenti" mediante le quali osservare criticamente la modernità. Ad esse fanno seguito quelle del sacro e del mistero. Nel sentimento della religiosità, nel mistero dell'infanzia, nelle rappresentazioni del sacro Gennari rintraccia possibili risposte, seppur problematiche, a quanto propone la società dell'apparenza e del mercato, della tecnocrazia e dei media.

Geld e Gewalt sono pronti a emergere: il denaro e il potere costituiscono due termini di riferimento vincolanti nella trattazione di Gennari. Possono essere rintracciati, in particolare, nella terza parte di Filosofia della formazione dell'uomo, dal titolo "Oggetto e soggetto". In essa i concetti s'inseguono in una drammaticità sequenziale che sembra continuamente dare e togliere energia vitale alle potenzialità dell'umano. I sistemi economico, sociale e scolastico s'intrecciano, mentre la riflessione sulla critica sociale di Horkheimer 
e Adorno si sviluppa in ulteriori passaggi che enfatizzano l'inessenza dell'attività del funzionario, del dominio e dell'autorità rispetto all'essenza della libertà, della formazione, dell'umano. Si scende poi negli interstizi della Legge e delle leggi per poter comprendere il rapporto fra diritto, scienza politica e filosofia della formazione. La politica, il politico e l'impolitico di Mann o l'agire strumentale e comunicativo di Habermas, nonché il distanziamento disincantato dall'oggetto e la presa di coscienza del nichilismo intrinseco alla crisi dell'Occidente dischiudono originali problematizzazioni pedagogiche.

Il testo non si ferma; deve ancora culminare nella quarta e più breve parte dedicata alla "Civiltà dell'uomo". La dura e drastica critica alla modernità disvela il proprio atto conclusivo in un serrato attacco all' "antiumanesimo" di Heidegger. La verità dell'essere e l'essenza dell'umano necessitano di uno "sprofondamento nell'umano" stesso, possibile per il tramite della formazione. È una formazione che articola $i$ propri significati rintracciandoli nelle "filosofie dell'umano", in grado di sondare la natura e il sentimento, la vita e il mondo riconducendoli a una formazione umana che è incontro, legame, fondazione ed anche trasformazione, libertà, mistero. Gennari delinea una "pedagogia dell'uomo fondamentale" che non include solamente tale "sprofondamento" nell'umano, poiché è possibile anche un tragico "sfondamento dell'umano". Gli interrogativi che lo studioso si pone e offre in maniera critica al vaglio interpretativo continuano a susseguirsi fino a quello conclusivo: "Che cosa è la pedagogia?». Abitare la domanda è interpretarla movendo dall'essenza e dal fondamento dell'uomo e di quella formazione che ognuno è. Per questo, essa rimane sospesa nel domandarsi dell'uomo e nella sua storica incapacità di formulare una risposta definitiva che proponga l'identificazione ontologica dell'uomo con la sua formazione.

Filosofia della formazione dell' uomo conclude così un lungo percorso d'interpretazione pedagogicamente impegnativo e filosoficamente rilevante. È un libro che offre alla pedagogia - anche attraverso una considerevole mole di indicazioni bibliografichefeconde concettualizzazioni e categorie, atte a interpretare con originalità i mondi della formazione dell'uomo. Lo fa proponendo la scelta dell'umano, in contrapposizione all'annientamento del medesimo, come unica possibilità per un uomo che "si forma davvero solo quando pensa, vive, conosce, filosofa" (p. 24), nonché "[...] ama, gioca e lavora potendosi "sentire" uomo" (p. 21). Di fronte alla tabe della Bildung e alla crisi del "principio di formazione", Mario Gennari indica come reagire: se non trascura la dignità del suo essere, l'uomo saprà ritrovare sé stesso proprio in quei processi che danno forma alla sua ineffabile e incommensurabile umanità.

ANNA KAISER

NÚÑEZ CUBERO, L. (2000) La escuela tiene la palabra. Temas educativos para la reflexión y el debate. Madrid, PPC.

Bajo este sugerente título, los lectores de esta obra tienen la oportunidad 
de aventurarse en un paisaje pedagógico rico en matices, encrucijadas y senderos cuyo recorrido, sin duda, conduce a un final de viaje que invita, en este caso, al lector no a instalarse en él, sin más, y contemplarlo como si de un mero turista se tratara, sino que le impele, por el contrario, a actuar con la "conciencia modificada" propia del viajero que se reconoce como parte misma de ese paisaje y, por ello, como parte misma de aquello que observa. El propio autor, el profesor Núñez Cubero, adopta en este libro esta particular "conciencia modificada" que proyecta igualmente al lector a lo largo de sus páginas. Esta "Conciencia modificada" permite al autor detenerse y profundizar en cuestiones claves que desde hoy se imponen a la educación y las instituciones que la promueven con toda la extensión que ambos términos comportan.

A lo largo de los siete capítulos que comprende la obra, sus reflexiones se detienen, en unos casos, en aspectos clásicos de la reflexión educativa - no por ello reiterativos o trasnochados en el tiempo- $y$, en otros, su punto de mira se centra en el análisis de los problemas educativos emergentes. En este sentido, la importancia de la educación, cuyo análisis combina con una ajustada reflexión de tintes prospectivos, la educación para la cohesión social, la educación para el desarrollo, la educación tecnológica y el vasto espacio de la educación en valores, jalonan el recorrido de esta obra que sirven, por otra parte, para reflexionar, con la mirada puesta en el presente, en uno de los problemas aún no resueltos en educación y que se concretan en aquello que hemos dado en calificar como "fracaso escolar".
Cada una de las cuestiones abordadas en este libro responden a un hilo conductor común que se constata en las respuestas pedagógicas que el autor infiere a la luz de los análisis y reflexiones elaboradas. Tras lo que podríamos identificar como un análisis de necesidades" educativas en la sociedad emergente -descritas en cada uno de los capítulos-, el profesor Núñez Cubero interpele con ello, tanto al propio sistema científico -la necesidad, subraya, de una visión interdisciplinar, de diseñar un espacio multidisciplinar para abordar la educación desde la complejidad- como al propiamente pedagógico que es, en definitiva, donde la escuela retoma su palabra.

Esta palabra se traduce, a la luz de las páginas de esta obra, en acciones racionalmente planificadas y, sobre todo - y unida a ellas - , se traduce asimismo - por lo que respecta al sistema educativo en su conjunto y sus agentes- en nuevos horizontes cognitivos, motivacionales, estratégicos y axiológicos, con los que aspirar, no a falaces ilusiones u horizontes irrealizables, sino al desarrollo de metas y funciones educativas irrenunciables.

La escuela tiene la palabra implica por ello pensar y actuar de acuerdo con criterios específicamente educativos; esto es, aquellos que redunden en una mejora de la calidad de vida de los ciudadanos y que optimicen la paradójica y ambivalente naturaleza de la condición humana. Estas metas y funciones irrenunciables requieren, según Núñez Cubero, la construcción de una escuela consistente que combata los efectos negativos del fenómeno que el propio autor ha categorizado con el término 
de "ruptura educativa". Esta escuela consistente es, por otra parte, una tarea irrenunciable en nuestro tiempo histórico donde la cohesión social y el pluralismo son condiciones necesarias -e ineludibles - para construir un espacio público común.

En definitiva, La escuela tiene la palabra comprende algo más que un recorrido por temas educativos para la reflexión y el debate, como podríamos obviamente deducir con el subtítulo de la obra. Además de todo ello, encierra toda una propuesta educativa pensada con grandes dosis de realismo desde y para el presente, por lo que los lectores - profesionales, investigadores y estudiantes- interesados en hallar claves precisas a los principales retos que se plantean hoy a la educación, encontrarán, sin duda, en este libro, explicaciones y análisis rigurosos a la par que sugerentes. Para quienes confiamos en la educación y sus agentes, aun conscientes que no todos los problemas sociales admiten, de modo exclusivo --Como parece deducirse hoy en día- una solución únicamente educativa, podemos felicitarnos de contar con esta innovadora obra que aborda cuestiones sobre educación de gran actualidad escrita, por otra parte, con un estilo ágil e inquisitivo, algo que sin duda agradecemos sus lectores.

Clara Romero Pérez

ORTEGA, P. y MÍNGUEZ, R. (2001) Los valores en la educación. Barcelona, Ariel.

Algunas veces, el conocido dicho de "Segundas partes nunca fueron buenas" se queda simplemente en eso, frase

(c) Ediciones Universidad de Salamanca tópica de conversación al uso cotidiano. La obra que proponemos lo pone claramente de manifiesto. A tal comentario contribuye la magnífica voluntad de estudio de sus autores que, después de ponernos sobre la pista de la auténtica "utilidad" del valor, amplían ahora el trazado - crucial e inevitable-de los valores en la educación, los enzimas del proceso educativo, como llegan a tildarlos en sugerente alocución.

Pero la ampliación de un trazado que se limitase a sumar paisajes sin mayor sustancia cognitiva acabaría con la paciencia lectora y provocaría, bien el abandono de la travesía, o bien la compulsiva aceleración revisora de las páginas que restaran. Muy lejos, fruitivamente lejos, de tal estado se encontrará quien se adentre en el volumen - uno de los mejores entre los miles de referencias generadas en torno al tópico- siempre, claro es, que le anime el deseo de saber más acerca de los valores y de su papel central en la teoría y la práctica de la educación, dentro y fuera de la escuela.

La mesura en el enfoque, el rigor no reñido con la fluidez expositiva, y la diáfana intención de enseñar al docente, al práctico que reflexiona, vías efectivas por las que encauzar su intervención, son aspectos a tener muy presentes en la decisión de recomendar un título en el vasto ámbito de la pedagogía. Este libro los cuenta entre sus activos y no se hace difícil proclamarlo a fin de animar su efectiva comprobación por parte de quien lo estime oportuno.

Lo que se ofrece al educador, al pedagogo, al profesor o, simplemente, a quien desee enterarse de lo que 
valen, realmente, los valores, de su dinámica en la construcción y optimización de los humanos, y de cómo influyen en la actuación de estos seres (de precaria inteligencia moral), es una aportación en toda regla pedagógica, esto es, la que no se limita a clarificar conceptos sino que ilustra y ejemplifica, con elegancia estratégica, exquisitos modos de auspiciar la competencia práxica del profesorado, agregando, de paso, oportunidades para el incremento de los beneficios socioafectivos que deben acompañar a los dispositivos de interacción cooperativa en las aulas o en otros contextos formativos.

La consideración filosófica y pedagógica de los valores no es, ni puede ser, exactamente la misma, sobre todo en el plano metodológico del discurso. No obstante, ambas consideraciones sí tienen que hacerse cómplices de idéntico anhelo, el de cimentar la arquitectura de la vida, de una vida con sentido en las coordenadas éticas de cada circunstancia personal.

En nuestro caso, cuenta el componente estable y cuenta, desde luego, el componente dinámico del valor. Son los condicionantes históricos marcas indelebles del valor y de su realización en un espacio y en un tiempo, con un anclaje cultural destilado en el fondo de un cuadro, cuyos bordes recuerdan, sutilmente, que el cambio perceptivo no anula la sustantividad axial de los grandes referentes axiológicos de la existencia humana.

Metidos en nuestro papel, el peligro aparece cuando las dudas se desparraman por doquier a la hora de hacer propuestas coherentes en o para los escenarios educativos. La contribución de los Drs. Ortega y Mínguez (Universidad de Murcia) advierte contra una relación entre pedagogía y valores basada en preceptos, cuando lo que se precisa son, justamente, principios para la acción, cuyo despliegue demanda una buena formación de los profesores en las estrategias más acordes con la razonable consecución de los objetivos curriculares y formativos en general.

Además, la acción que mentamos será estratégica en el sentido de poder prever, y disponer, adecuadamente los raíles de una educación para la democracia, cuidando de no caer en la atávica conformación de "virtudes patrias", exaltadoras de lo que separa y no de lo que une. Una pedagogía de los valores o es una pedagogía del encuentro, o no es más que un sucedáneo de pedagogía, indigna de ser nominada como tal en el concierto de las razones sobre el progreso humano. Será, pues, una pedagogía del interés común, en la que los matices no han de erosionar la significatividad del propósito, ni donde el ideal relacional pueda acabar convertido en ceguera ante lo intolerable.

En una sociedad red, digitalizada, abierta, con las mejores posibilidades de comunicación que jamás se hayan dado, no hay justificación para que se haya descuidado el respeto a los valores fundamentales, a los derechos básicos, que son reales y no fantasiosos. He ahí una gran tarea del educador: mostrar cómo manda esa realidad concreta del valor en cada lugar, el modo en que determina formas de pensar y de actuar, muchas veces mitificadas, alejadas de las necesidades reales de la vida cotidiana, de las virtudes cívicas, en cuyo edificante desarrollo nos jugamos 
bastante de nuestro bienestar, de la posibilidad de ser con los otros seres.

Todo esto apunta a la conveniencia, práctica, de como movilizar la mejor educación posible de los valores, sabiendo perfectamente que el cometido se escapa a cualquier digresión convencional sobre la responsabilidad profesional de un docente, o sobre la misma exigencia que cabe hacer a la institución escolar.

La experiencia escolar es sólo una parte -importante sí pero sólo unade la experiencia vital de los sujetos. Por ello habrá que aprovechar en los centros educativos para intentar proyectar la experiencia del valor. Lo que importa, de verdad, es la apropiación del valor de justicia, de libertad, de tolerancia, de solidaridad, o de lo que se tercie, cuando la persona se implica en una acción o tarea que le interesa o que sirve a su crecimiento como sujeto. A tal efecto, buen monto de prácticas, para escoger a gusto de necesidades y situaciones, es el que tenemos a nuestra disposición en el volumen, que predica con el ejemplo a la hora de mostrar una auténtica complementación entre teoría y práctica, siempre necesaria pero vital en el tema que al que nos convocan.

Ya vamos terminando. La crítica del ensayo, y más si éste es producto de la buena incursión investigadora, no puede discurrir únicamente por veredas de racionalidad técnica, según sea, o le parezca al autor o autores que sea, la conjunción de elementos formales que lo convierten en apto para el consumo intelectual en un determinado campo. La senda de una expresividad más llamativa y abierta al lado emocional del sujeto en cuyo marco de vida se representa un tópico formal, debe despejarse definitivamente si queremos que los libros ayuden a enriquecer la peripecia lectora. Para ello, nada mejor que dar cabida a la experiencia narrada, al relato de cómo influyen los grandes conceptos en la toma de contacto con el mundo, o al revés, de cómo la inmersión en un medio físico y cultural modula nuestra orientación narrativa de las cosas y de los acontecimientos.

Lo bueno es que la educación en valores permite - casi lo exige- hacer esto. La única precaución es la de no caer en la tentación de admoniciones abstractas para lo que han de ser ejercicios muy prácticos, sin que falten los juegos que enganchen a niñas y niños en dinámicas de comunicación reactiva ante dilemas morales, o ante simples situaciones de la vida cotidiana con implicaciones ético-cívicas, y cuya demanda de análisis y toma de decisiones comprometidas se hacen más patentes en época de incertidumbres como la que vivimos.

Nada de ello es ajeno a las páginas de un libro como éste, indisociable de una trayectoria investigadora sólida y bien conocida en la pedagogía española. Y es justo reconocer la forma en que se ha ido imprimiendo carácter al estudio de los valores en la educación, no pocas veces objeto de superficial repaso en publicaciones un tanto prosaicas.

Por algún lugar de la buena crítica literaria escucho el susurro de que el valor de una obra no procede ya de su belleza, de la novedad que introduce en el mundo o de la incapacidad de iluminar lo oscuro, sino de su representatividad 
social o cultural. Concuerdo bastante con esta apreciación y me sirvo de ella para reiterar lo básico: que estamos ante una aportación genuinamente representativa de un ámbito de estudio central a la pedagogía, y que ningún educador debería desperdiciar la oportunidad que estas páginas le brindan de mejorar su utillaje axiológico, condición necesaria si de lo que se trata es de optimizar la misma intervención pedagógica.

Miguel A. Santos Rego

ORTEGA RUIZ, P. y MÍNGUEZ VALLEJO, R. (2001) La educación moral del ciudadano de boy. Barcelona, Paidós.

Los autores, reconocidos en nuestro ámbito pedagógico por su buen quehacer en la rica veta de la educación y valores, aportan en esta ocasión un excelente trabajo teórico-práctico acerca de dos temas de renovada actualidad, que ya en el título de su obra aparecen fundidos: la "educación moral"... del "ciudadano de hoy".

El contenido del libro - bien prologado por el profesor J. C. Mèlich- se vertebra en cuatro sustanciosos capítulos: uno introductorio, en el que se cuestiona con profunda reflexión el sentido que debe tener hoy la educación moral, así como su vinculación con una genuina educación de la ciudadanía; a ese primero le siguen tres más, en los que se abordan, respectivamente, la triple competencia moral que precisa un cultivo especial en nuestro contexto actual: "competencia moral para un diálogo inter-cultural”, "...para una relación entre Norte y Sur", y "...para la

(c) Ediciones Universidad de Salamanca protección de un medio natural y urbano". Una cabal conclusión cierra este magnífico escrito, utilísimo para los teóricos de la educación (en orden a renovar esquemas básicos en un tema de tanto bulto) y para los prácticos educadores (al dotarles de sugerencias concretas a la hora de iniciar tan importante educación en su tarea cotidiana).

Sería ingenuo intentar reseñar aquí lo más esencial siquiera del rico contenido encerrado en el libro ahora comentado. Parece más oportuno traer a colación algunas tesis de los mismos autores, e intentar glosarlas en determinados momentos. En el primer capítulo, por ejemplo, afirman: "educar moralmente es educar para la responsabilidad, para responder no sólo al otro, sino del otro" (p. 24); porque "ser hoy una persona educada moralmente y un buen ciudadano, significa no vivir de espaldas o indiferente a los graves problemas que nos afectan a todos; de modo que los problemas del otro empiecen a ser nuestros problemas" ( $\mathrm{p}$. 28). Afirmaciones, éstas, que fluyen de algunos principios éticos que Ortega y Mínguez hacen muy propios: la dimensión heterónoma de la ética (Levinas), de donde brota la inevitable respuesta solícita, solidaria y compasiva a la "llamada" de todo otro; el compromiso con toda realidad personal y sociocultural ajena concreta, real, viva. Ese marco ético - aquí sólo apuntado- es el que legitima, coherentemente, las propuestas pedagógicas genéricas y concretas de los autores en las restantes páginas que componen su libro.

La "competencia ético-moral", en efecto, no se agota en "el simple aprendizaje de unas estrategias y habilidades" necesarias en este terreno, pues "sin la 
experiencia de los valores (morales) no es posible su aprendizaje". Sí; en la escuela, en la familia, o en cualquier otro contexto educativo, "se hace indispensable la referencia a unos modelos que, de un modo relativamente estable y continuado, constituyan una experiencia del valor moral deseado para que éste pueda ser aprendido" [...], porque el niño-adolescente (a diferencia, en parte, de lo que puede ocurrir en el plano de lo cognitivo) no aprende una conducta valiosa independientemente de otra persona que la realice" (pp. 3545). La "responsabilidad" pues, por un lado, y la "experiencia" de los valores, por otro, se apuntan insistente y convincentemente como dos axiomas insoslayables en toda educación moral que se precie de ser tal.

Sentados estos presupuestos, los autores conforman los tres capítulos siguientes a modo de aplicaciones específicas de los mismos. Así, el segundo de ellos se centra en la "educación intercultural". Tras abordar tópicos básicos, tales como el componente cultural del hombre concreto, o el resbaladizo tema del relativismo-universalismo, Ortega y Mínguez se dedican a desentrañar lo más esencial de la tan llevada y traída educación intercultural. Así, "las muestras de intolerancia no van dirigidas con una cultura determinada, sino también (¿y fundamentalmente?) contra las personas que las protagonizan"; $\mathrm{O}$, en una versión ahora positiva, "la educación intercultural no pretende sólo el respeto a las ideas y creencias del otro diferente..., sino además el reconocimiento de su dignidad, de mi responsabilidad hacia él, quien por su sola presencia demanda de mí una respuesta, no sólo de comprensión «intelectual" de su cultura, sino de acogida de su persona. Tal respuesta es un acto ético..., por lo que la educación intercultural, si es tal, se traduce necesariamente en una educación moral. (p. 66). Poco se ha dicho tan acertado - al menos en nuestro contexto próximo- sobre lo que debería constituir el alma misma de la educación intercultural, tan centrada todavía en el cultivo romántico de las diferencias adjudicadas a las culturas, tan abstractas éstas como desencarnadas.

El tercer capítulo se entiende con la "Competencia moral para una relación entre Norte y Sur". Aquí, los autores desgranan toda una serie de tesis capaces de fundamentar un ámbito como éste, con frecuencia demasiado banalizado. "Las relaciones de los pueblos Norte-Sur se han visto desde la perspectiva de la cooperación [...]; pero se han olvidado que no toda cooperación lleva al desarrollo, promoción y liberación de los países pobres; que hay formas de cooperación que prolongan la situación de dependencia" de estos pueblos. Las relaciones con éstos, ciertamente, "no se deberían fundamentar en la oportunidad política de cooperar, sino en la exigencia moral del reconocimiento de una deuda pendiente" (pp. 104-105); es decir, una "deuda" contraída contra la dignidad de los que sufren injustamente la inmoralidad cotidiana - y política- de quienes están en mejor posición. Algo muy bien expresado por los autores así: "La ayuda a los pueblos del Sur [...] implica necesariamente el reconocimiento de los otros de su inalianable condición de personas, de su situación de seres bumanos desposeidos de aquello a lo que tienen derecho" (p. 106). Desde estos parámetros resulta inteligible una 
nueva conceptualización de términos clave en este campo, tales como la solidaridad, la justicia o la misma cooperación. Desde esa óptica, también, queda resaltada la necesidad de una educación moral basada en la genuina compasión: es necesario apostar - comentan ellos"por un nuevo tipo de educación, que asuma el sufrimiento ajeno como algo injusto, que haga de la responsabilidad frente al otro, y del compromiso de actuar, una cuestión irrenunciable" ( $p$. 121). Una educación para la empatía, la prosocialidad y el consumo - como se apunta de forma práctica en páginas posteriores - tienen en ese marco todo su profunda razón de ser.

El cuarto y último capítulo se vuelca en la "Competencia moral para la protección del medio natural y urbano". En estas páginas pueden encontrarse pistas de gran valía para entender con hondura de sentido conceptos tan trillados como, por ejemplo, el desarrollo sostenible. Valga, como síntesis, esta cita: "A pesar de las declaraciones solemnes sobre la preservación del patrimonio natural, la filosofía del consumo y despilfarro, del crecimiento ilimitado aún sigue orientando las políticas de los países desarrollados, con la pretensión, además, de imponerla a los que aún están en vías de desarrollo. (Ahora bien), sin un desarrollo sostenible que satisfaga las necesidades de las actuales generaciones, sin comprometer la capacidad y necesidades de las futuras, no es posible imaginar un planeta a la medidad del hombre. Esto implica cambiar la filosofía de fondo que hasta ahora ha explicado las relaciones del hombre con su medio natural" (p. 161). La educación en esta nueva perspectiva se presenta como algo esencial; una educación moral que ayude a "asumir la responsabilidad frente a los demás desde el convencimiento de que hay cosas que no son de uso exclusivo, sino que pertenecen a todos" (p. 167).

El libro aquí comentado tiene la virtualidad de ofrecer claves novedosas para los pedagogos, en general, y particularmente para los interesados en la educación moral y en cada uno de los tres grandes ámbitos a la que ésta se aplica. Su interés dimana también de la utilidad práctica que adquieren sus propuestas pedagógicas concretas a la hora de trabajar en la realidad educativa estas dimensiones morales que están - con toda razón- en el centro de la sensibilidad de todo ciudadano de hoy, como acertadamente apunta el título mismo del libro.

Me felicito de poder haber leído con reposo esta obra de los profesores Ortega y Mínguez, con ocasión de su recensión, a la vez que invito a todos aquellos interesados en los temas que aborda a leerla también, tanto para una mayor formación como para rescatar pistas utilísimas para su puesta en acción.

José Antonio Jordán Sierra

QUINTANA CABANAS, J. M. a (2001) Las creencias y la educación. Pedagogía cosmovisional. Barcelona, Herder.

Como continuación y ampliación a su obra La axiología como fundamentación de la filosofía (Madrid, UNED Ediciones, 2000) el profesor Quintana Cabanas nos ofrece en este libro su propuesta de una educación cosmovisional. 
Por tal entiende el ayudar al individuo a que se oriente en sus creencias, buscando con interés y sabiduría el sentido del mundo y de la vida y, como resultado, formándose una cosmovisión que le permita situarse en el mundo y llevar una existencia capaz de colmar sus aspiraciones humanas más profundas". No hay hombre - y por extensión educación- sin cosmovisión. En este sentido la educación cosmovisional coincide con la "educación trascendente", si bien se diferencia de la pedagogía estrictamente religiosa porque sus propuestas son de tipo confesional y nuestro autor pretende situarse en un momento anterior, previo y condicionante. De alguna manera la pedagogía cosmovisional constituye una base para la pedagogía religiosa ya que las creencias y los valores no han de darse al final del conocimiento sino al principio. De modo que sólo si se tiene una cosmovisión trascendente está justificada la educación religiosa.

Quintana es pionero en España -en Alemania ya lo ha hecho Brezinka - en proponer el tema de la pedagogía cosmovisional como una nueva rama pedagógica con futuro que aborda los aspectos filosófico-teoréticos de la educación cosmovisional que es aquella que atiende a la educación trascendente de la persona. Al promocionar una pedagogía humanista en la que convergen la ontología y la axiología, Quintana defiende la importancia de las creencias tan denostadas por el pensamiento moderno. Nuestro autor parte, pues, de una valoración positiva de las creencias que constituyen un valor y un enriquecimiento de la persona. Asimismo lamenta que muchos jóvenes pierdan la confianza en las creencias en las crisis de crecimiento de la adolescencia. De ahí la importancia de la educación porque las creencias que tienen historia no brotan en el individuo por generación espontánea sino gracias a la educación que se da siempre en un contexto cultural determinado: las creencias se aprenden o no se tienen. Frente al relativismo posmoderno que equipara a todas las culturas y religiones, Quintana sostiene que no todas son iguales. Su posición es clara: aunque todas las culturas $-\mathrm{y}$ lo mismo sirve para las religiones-cumplen con la misma función social, no todas lo hacen con idéntica calidad desde el punto de vista humanista.

El profesor Quintana que estudió en Munich junto al profesor Reinhard Lauth durante el año 1968, se familiarizó con este tipo de pensamiento de carácter espiritual e idealista que, en su caso, entronca con la filosofía perenne y se abre al mundo de los valores. En realidad para Lauth el problema del sentido es el problema central de la vida humana que en última instancia depende de la cosmovisión que cada cual tenga. La adjudicación de un sentido al mundo es una cuestión de creencia, de fe. De ahí que el profesor Quintana aúne lo racional y lo valorativo en una síntesis en la que germina la noción de sabiduría que viene a ser una virtud moral con base intelectual. Además Quintana confía en la sabiduría como facultad de las creencias y de los valores. Así la sabiduría (phronesis en griego, prudentia en latín) viene a ser una combinación de inteligencia, razón y experiencia que permite dirigir la vida de la mejor manera posible, aplicándose a formar 
la cosmovisión, a conocer y elegir los valores y a regir la acción moral. Pero aún hay más porque la sabiduría se funda en un saber adquirido no en el estudio, sino en una reflexión prolongada sobre las experiencias de la vida. El sabio procede por intuición sintética y no siguiendo el método analítico y discursivo del científico, lo cual significa que la persona opta por su cosmovisión a través de la sabiduría.

La cuestión del sentido supera los estrictos límites del modelo científico que recurre a la "verdad de los hechos" porque la mente intuye emocionalmente el sentido. De ahí que la idea de sentido sea para el hombre cierta pero no evidente con lo cual el sentido del mundo -negado por muchos autores- no está claro. Pero Quintana insiste en lá necesidad de hallarlo -el hombre es un ser que se abre al sentido- y verlo en un horizonte trascendente. La única solución radica, pues, en que él mismo quiera verlo, lo afirme y lo reconozca porque - de acuerdo con Lavelle- -el mundo no tiene sentido por sí mismo: somos nosotros quienes le damos un sentido". En sintonía con lo dicho, Quintana - que frente a la tesis de que los valores dan fines $y$, por tanto, sentido, defiende un axilogismo según el cual son los fines los que dan sentido $y$, por ende, determinan valores - confirma la existencia del sentido de la vida que se expresa en los valores. Entre ontología y axiología, Quintana aboga por la prioridad de la primera por más que ser y valor se condicionen recíprocamente. De modo que para Quintana la persona dará sentido a su vida a través de los fines y, a partir de ellos, adoptará los valores correspondientes.

Es sabido que la educación en las creencias - y especialmente en las religiosas- ha constituido uno de los grandes debates de la pedagogía contemporánea. Han sido frecuentes - Natorp, Ferrer y Guardia, Neill, Russell, Skinner, Suchodolski- los adversarios de una educación trascendente. Quintana se adhiere a los defensores de una educación abierta a la trascendencia que, respetando el pluralismo de las sociedades modernas, permita avanzar en la educación en las creencias. En consecuencia, aboga por una educación de la religiosidad que es mucho más que una función socializadora o moralizadora. Es obvio que una de las batallas escolares que más polvareda ha levantado - y continuará levantando próximamente- es la educación en las creencias. Mientras para unos las creencias han de desaparecer de la escuela otros defienden la educación confesional. Existen soluciones intermedias, pero todo da a entender que la cuestión - en una sociedad abierta a la recepción de otras culturas y religiones- no está zanjada. La solución que plantea Quintana intenta superar aquellas viejas polémicas (escuela laica, neutra, pluralista, confesional, atea), señalando que la religiosidad es un rasgo natural del hombre no siendo en modo alguno sinónimo de oscurantismo, sino auténtico humanismo dado que el hombre es un ser religioso (Zubiri). Desde esta perspectiva la educación cosmovisional viene a ser una pedagogía pre-religiosa que abre el horizonte de la transcendencia a fin de dar sentido a la vida humana. La conclusión 
del libro que nos ocupa es clara: la educación debe enseñar al hombre a formarse una cosmovisión -o lo que es lo mismo, un conjunto de creenciasen la que tenga cabida esa dimensión trascendente que es inherente al ser del hombre.

\section{Conrad Vilanou}

SANTOS REGO, M. A. y LORENZO MOLEDO, M. M. M. (eds.) (2001) A construcción educativa da cidade. Unba perspectiva transversal. Santiago de Compostela, Universidade de Santiago de Compostela.

La presente publicación se sumerge en una insinuante temática socioeducativa, dentro del ámbito de la educación no formal, esto es, la valoración de la ciudad desde una mirada educativa. Por tanto, educación y ciudad constituyen dos importantes pilares que interaccionan para convertirse en un reto, la ciudad educadora.

La obra expone la ciudad como un ámbito adecuado para el desarrollo de aprendizajes cívicos, favoreciendo también la vida cognitiva y afectiva de los ciudadanos. En ella se cuestiona la función de la ciudad y para ello, cuenta con la colaboración de un grupo de expertos preocupados por defender una concepción transversal de la ciudad, con el fin de asentar y mejorar la calidad de vida de las personas que habitan en los diversos municipios, además de entender que la ciudad educadora se integra dentro de una educación política o de una educación cívica-moral.
El libro se compone de tres partes. En la primera, desarrollada por Miguel A. Santos Rego y M. ${ }^{a}$ do Mar Lorenzo Moledo, editores de la publicación, se proyecta una visión teórica sobre la ciudad, "Pedagoxía e cidade: a permanencia dun clásicom. Implican en el proceso de estudio el término de educación en la ciudad, reflexionando sobre el significado de ciudad educadora e incidiendo en su dimensión cultural.

Bajo el título de "Ciudadanía y educación: el desarrollo de nuevos modelos socioeducativos", Antoni J. Colom Cañellas, de la Universidad de Baleares analiza el papel que juegan las entidades locales al fundirse términos como política, educación y sociedad civil para establecer las bases de la pedagogía urbana. Asimismo, Xosé Luis Ba-rreiro Rivas, de la Universidad de Santiago, profundiza en los conceptos de ciudad y ciudadano desde ópticas culturales y políticas. Asimismo, resalta la necesidad de hablar de nuevas dimensiones de ciudadanía y entiende la ciudad como una escuela de valores y actitudes.

"Educación y ciudad sostenible", es la denominación que concede a su exposición José A. Cuerda Montoya, ex alcalde de la ciudad de Vitoria. Destaca la visión de la ciudad como espacio social, urbano y comunidad de valores.

La perspectiva filosófica defendida por Javier Echeverría y rotulada "Educar para la ciudad telemática" es un claro exponente de los actuales y necesarios campos de aprendizaje y transmisión de conocimientos a través de las nuevas tecnologías de la información y de las telecomunicaciones.

La segunda parte de esta publicación abarca cuatro capítulos que intentan 
construir un proyecto cívico de ciudad. El primero, "Memoria e identificación da cidade" por el escritor Suso de Toro, es un análisis de su memoria y vivencia de la ciudad, identificándola en la actualidad como un caos. El segundo corresponde al interesante trabajo de Xerardo Estévez, "Educación e urbanismo: a cidade como proxecto cívico", un enfoque arquitectónico para comprender la ciudad contemporánea. Los capítulos tercero y cuarto se centran respectivamente en dos referencias básicas a imitar, la rehabilitación de la villa ourensana de Allariz, expuesta por su alcalde Anxo Quintana, "Educación, desenvolvimento local e solidaridade" y la ciudad santiaguesa, "Compostela: unha cidade educadora. Presente e futuro" una visión pedagógica hecha realidad, desde el Departamento de Educación del Ayuntamiento de Santiago de Compostela, por Xosé M. Rodríguez-Abella.

La tercera parte del libro, bajo el epígrafe -Educación e ciudadanía. A dobre vertente política-, aunque centrado en la ciudad compostelana, se convierte en un espacio de reflexión y acercamiento a las distintas redes de expresión de la sociedad civil (Administración local, movimiento cultural y asociacionismo vecinal y función de los principales representantes políticos gubernamentales).

A modo de anexo se insertan lecturas como la Carta de ciudades educadoras, que ya ha cumplido una década desde su promulgación; los Documentos Complementarios y el Listado de Ciudades Educadoras. Todo ello con el ánimo de facilitar información adicional

(C) Ediciones Universidad de Salamanca a cuantos se sientan interesados por estos temas.

No cabe duda que el libro resulta de gran utilidad para aquellos docentes y profesionales que velen por estilos educativos saludables. Prueba de ello es la conjunción de expertos aquí reunidos por una misma causa, armonizar las vivencias transcurridas en las ciudades potenciando climas de ciudadanía y calidad de vida.

Carmen Pereira Domínguez

SORIANO DÍAZ, A. (2001) Maltrato infantil. Madrid, San Pablo.

El maltrato a la infancia no es un fenómeno nuevo ni reciente, muy al contrario, éste se viene produciendo desde los comienzos de la humanidad. Ésta es la razón por la que en el primero de los capítulos de este libro se realiza un recorrido de carácter histórico en el que se plasman, de forma resumida y sintética, las situaciones de maltrato por las que la infancia ha ido atravesando a lo largo del acontecer histórico de la humanidad.

En el capítulo segundo "Qué es el maltrato infantil: tipos", se profundiza en el conocimiento de este campo. Para ello, el autor comienza aclarando qué entiende por este fenómeno. Posteriormente, define los distintos tipos de malos tratos que existen asociados a sus correspondientes manifestaciones o indicadores.

Nunca se maltrata por azar. Siempre existen razones que hacen que se llegue a estas situaciones. En el capítulo tercero, "Por qué los adultos maltratan a 
los niños", se realiza un recorrido sobre las distintas y más importantes perspectivas que intentan dar explicación a este fenómeno prestando especial atención a la perspectiva ecosistémica.

En el siguiente capítulo "Los niños se hacen mayores: adultos que fueron maltratados en su infancia", se dan a conocer algunos de los problemas que, debido a un pasado de malos tratos en la infancia, pueden acarrear los adultos a lo largo de su vida. Además se proponen soluciones y estrategias para resolver esta problemática.

Los esfuerzos realizados por proteger a la infancia más desvalida han derivado de los cambios de concepción que, con respecto a la infancia, se han ido produciendo a lo largo de la historia del hombre. Todo ello ha quedado plasmado en la legislación protectora de menores. Es importante conocer la existencia de esta legislación y, sobre todo, de algunas leyes específicas, al menos en un nivel básico. En el quinto de los capítulos, «Marco legal: legislación protectora de la infancia", se realiza un recorrido sobre el marco legal más sobresaliente que, en materia de protección de menores, existe en la actualidad en el ámbito internacional y, fundamentalmente, en el Estado español.

La Administración pública está obligada a ejercer la acción protectora sobre los menores que han sido objeto de malos tratos, o bien se encuentran en situación de riesgo o desprotección sociofamiliar. Para ello, dispone de una serie de recursos que hacen posible dar respuesta a las necesidades que se plantean. En el capítulo sexto "Qué se hace para prevenir y atender a la infancia en situación de riesgo sociofami- liar", se presentan los recursos de atención especializados con que cuenta la Administración pública.

Para aquellos lectores que quieran ampliar o profundizar en el conocimiento del problema de los malos tratos a la infancia, en el último apartado del libro, "Anexo", se muestran una serie de recursos tales como direcciones de organismos protectores de la infancia, listados de asociaciones específicas, fuentes de información de carácter internacional y nacional, publicaciones de interés, etc.

Al comienzo de cada uno de los capítulos expuestos aparecen unas "cuestiones previas" que intentan motivar la lectura de los mismos, a la vez que ayudan a interrogarse sobre aspectos concretos de cada uno de ellos. Al final de cada uno de estos bloques se ha colocado una "propuesta de actividades" que ayudará a profundizar, clarificar y sintetizar el contenido de los textos.

José Álvarez Rodríguez

SUBIRATS I HUMET, J. (coord.). Gobierno local y educación. La importancia del territorio y la comunidad en el papel de la escuela. Barcelona, Ariel.

En una sociedad globalizada y mundializada, donde los procesos y la manera de pensar global es la que impera, rescatar la importancia y valía del territorio en cuestiones que afectan directamente al mundo educativo es, además de una tarea compleja, una preocupación de vital importancia. La inquietud por el engranaje y cohesión 
entre educación y territorio es la que ocupa el sentido y finalidad de los autores de este libro, más concretamente, el lugar que debe ocupar la educación dentro de las políticas públicas de cara a asumir los retos y expectativas que se van fraguando y desencadenando. Frente a una concepción formativa que conlleva fragmentación y segmentación, plantean el debate educativo en términos de diversidad, pluralismo, acuerdo social, flexibilidad y calidad educativa, proponiendo políticas de fuerte contenido transversal y con una consistente presencia local.

El libro se estructura en seis capítulos; los tres primeros de carácter más teórico y los siguientes con un marcado sentido práctico y de actuación. En el primer capítulo, se analiza el papel que el territorio ha jugado y juega actualmente en la configuración social y educativa de las comunidades locales, incidiendo en la necesidad de apostar por los espacios cotidianos, la relación cara a cara y la interacción diaria como fomentadores del desarrollo local y, por consiguiente, de los procesos formativos, analizando el concepto de comunidad y su relación con el de escuela, proponiendo al final el constructo de "la escuela comunidad". En el segundo se atiende al estudio de la educación en el marco de los servicios personales locales, mostrando cómo debe entenderse el concepto de servicios locales y cuál debe ser el ámbito de la educación en ese espacio. Para ello, por un lado, definen los servicios personales locales, sus particularidades y, por otro, analizan cuál debe ser el desarrollo de los servicios personales en el ámbito local mediante un estudio de caso, desde lo que denominan dimensiones conceptuales, sustantivas y operativas. Y en el tercero se muestra un análisis puramente transversal del ámbito local de la educación, incluyendo aportaciones teóricas y presentando el proceso de la elaboración de los mapas de la ciudad y el gobierno de los mismos como instrumentos que permiten conocer el flujo formativo local y la consiguiente acción integrada de los gobiernos locales.

Posteriormente, en el capítulo cuarto, se analizan brevemente las realidades de los sistemas educativos de algunos países europeos en lo que atañe al papel de los gobiernos locales, permitiendo así examinar nuestra situación actual y poder plantear líneas de intervención más adecuadas desde las distintas administraciones educativas de nuestro país. A continuación, en el capítulo quinto, se realiza un análisis jurídico de las competencias locales y su autonomía en relación al campo educativo, incidiendo en los contenidos más relevantes de nuestra legislación y, presentando, por un lado, el papel de la enseñanza en la legislación de régimen local y en la legislación educativa $y$, por otro lado, el rol que juegan los entes locales en la titularidad de los centros docentes y el personal docente y no docente. En el último capítulo, a partir de un somero análisis de la situación de la sociedad actual - cambios económicos, tecnológicos, en valores, institucionales, de la escuela-, sus retos y necesidades, presentan las oportunidades educativas que tienen los "Proyectos educativos de ciudad", que definen los autores como 
"Conspiración educativa de la ciudadanía" desde un estudio concreto.

En líneas generales estamos ante un trabajo en el que se defiende que "la gente del sitio es la que está en mejor situación para identificar los problemas y los retos a los que se enfrentan las escuelas" y donde se plantea la necesidad de avanzar hacia políticas educativas descentralizadas, prescindiendo de planteamientos homogeneizadores. Todo ello desde enfoques interdisciplinares y cooperativos entre profesionales del campo jurídico, político, económico y, como no, educativo. Se trata, por tanto, de un libro útil tanto para quienes están actuando en esferas centrales y locales del ámbito educativo, como para los profesionales docentes, padres, educadores y todos aquellos interesados por la lucha contra la exclusión, como los mismos autores señalan, argumentando que, la educación, "es una fuente indispensable de construcción de las específicas identidades individuales".

José Manuel MuÑoz RodRíGuez 